\title{
On Certain Multiple Bailey, Rogers and Dougall Type Summation Formulas
}

\author{
By
}

\author{
Jan F. VAN DIEJEN*
}

\begin{abstract}
A multidimensional generalization of Bailey's very-well-poised bilateral basic hypergeometric ${ }_{6} \psi_{6}$ summation formula and its Dougall type ${ }_{5} H_{5}$ hypergeometric degeneration for $q \rightarrow 1$ is studied. The multiple Bailey sum amounts to an extension corresponding to the case of a nonreduced root system of certain summation identities associated to the reduced root systems that were recently conjectured by Aomoto and Ito and proved by Macdonald. By truncation, we obtain multidimensional analogues of the very-well poised unilateral (basic) hypergeometric Rogers ${ }_{6} \phi_{5}$ and Dougall ${ }_{5} F_{4}$ sums (both nonterminating and terminating). The terminating sums may be used to arrive at product formulas for the norms of recently introduced $(q$-) Racah polynomials in several variables.
\end{abstract}

\section{§1. Introduction}

In this paper certain multidimensional generalizations are studied of the summation formulas

$$
\sum_{\lambda \in Z} \frac{\Pi_{r=1}^{4}\left(q^{1+g_{r}+z+\lambda}, q^{1+g_{r}-z-\lambda} ; q\right)_{\infty}}{\left(q^{1+2 z+2 \lambda}, q^{1-2 z-2 \lambda} ; q\right)_{\infty}}=\frac{(q ; q)_{\infty} \Pi_{1 \leq r<s \leq 4}\left(q^{1+g_{r}+g_{s}} ; q\right)_{\infty}}{\left(q^{1+g_{1}+g_{2}+g_{3}+g_{4}} ; q\right)_{\infty}}
$$

(with $2 z \notin\left(Z+\frac{2 \pi}{i \log q} Z\right)$ ) and

Communicated by Y. Ihara, January 6, 1997.

1991 Mathematics Subject Classification. Primary: 33D20; Secondary: 33C20, 11 L05.

Key words and phrases. very-well-poised (basic) hypergeometric series in several variables,

Bailey and Dougall type bilateral summation formulas, Rogers and Dougall type unilateral summation formulas.

Work supported in part by the Natural Sciences and Engineering Research Council (NSERC) of Canada.

* Centre de Recherches Mathématiques, Université de Montréal, C.P. 6128, succursale Centre-ville, Montréal (Québec), H3C 3J7 Canada. 


$$
\sum_{\lambda \in \mathbb{Z}} \frac{\Gamma(1+2 z+2 \lambda) \Gamma(1-2 z-2 \lambda)}{\Pi_{r=1}^{4} \Gamma\left(1+g_{r}+z+\lambda\right) \Gamma\left(1+g_{r}-z-\lambda\right)}=\frac{\Gamma\left(1+g_{1}+g_{2}+g_{3}+g_{4}\right)}{\Pi_{1 \leq r<s \leq 4} \Gamma\left(1+g_{r}+g_{s}\right)}
$$

(with $2 z \notin Z Z Z$ ), where it is assumed that $0<q<1$ and $\operatorname{Re}\left(1+g_{1}+g_{2}+g_{3}+g_{4}\right)>0$. (For conventions regarding the notation, we refer to the remark at the end of this introduction.) The conditions on $z, q$ and on the parameters $g_{1}, g_{2}, g_{3}$, $g_{4}$ guarantee that the terms on the 1.h.s. are finite and, moreover, that the series converge in absolute value. The sum in (1.1a) is (for generic $z$ ) equivalent to Bailey's very-well-poised bilateral ${ }_{6} \psi_{6} \operatorname{sum}[\mathrm{B}, \mathrm{GR}]$

$$
\begin{aligned}
& \sum_{\lambda \in \mathbb{Z}} q^{\left(1+g_{1}+g_{2}+g_{3}+g_{4}\right) \lambda}\left(\frac{1-q^{2 z+2 \lambda}}{1-q^{2 z}}\right) \prod_{1 \leq r \leq 4} \frac{\left(q^{z-g_{r}} ; q\right)_{\lambda}}{\left(q^{1+g_{r}+z} ; q\right)_{\lambda}}
\end{aligned}
$$

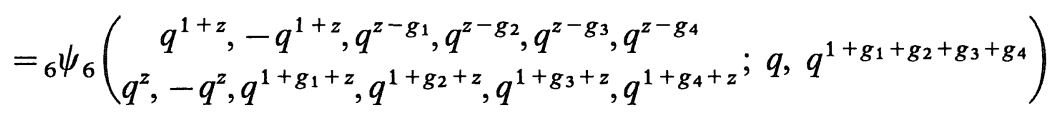

$$
\begin{aligned}
& =\frac{\left(q^{1+2 z}, q^{1-2 z} ; q\right)_{\infty}}{\Pi_{r=1}^{4}\left(q^{1+g_{r}+z}, q^{1+g_{r}-z} ; q\right)_{\infty}} \frac{(q ; q)_{\infty} \Pi_{1 \leq r<s \leq 4}\left(q^{1+g_{r}+g_{s}} ; q\right)_{\infty}}{\left(q^{1+g_{1}+g_{2}+g_{3}+g_{4}} ; q\right)_{\infty}}
\end{aligned}
$$

$(0<q<1)$ and the sum in (1.1b) corresponds to the degenerate case $q \rightarrow 1$, which amounts to Dougall's very-well-poised bilateral ${ }_{5} H_{5}$ sum [Do, B]

$$
\begin{gathered}
\sum_{\lambda \in \mathbb{Z}}\left(1+\frac{\lambda}{z}\right) \prod_{1 \leq r \leq 4} \frac{\left(z-g_{r}\right)_{\lambda}}{\left(1+z+g_{r}\right)_{\lambda}} \\
={ }_{5} H_{5}\left(\begin{array}{c}
1+z, z-g_{1}, z-g_{2}, z-g_{3}, z-g_{4} \\
z, 1+z+g_{1}, 1+z+g_{2}, 1+z+g_{3}+1+z+g_{4}
\end{array} ; 1\right) \\
=\frac{\Pi_{r=1}^{4} \Gamma\left(1+g_{r}+z\right) \Gamma\left(1+g_{r}-z\right)}{\Gamma(1+2 z) \Gamma(1-2 z)} \frac{\Gamma\left(1+g_{1}+g_{2}+g_{3}+g_{4}\right)}{\Pi_{1 \leq r<s \leq 4} \Gamma\left(1+g_{r}+g_{s}\right)}
\end{gathered}
$$

(with the same convergence condition on the parameters $\operatorname{Re}\left(1+g_{1}+g_{2}+g_{3}+g_{4}\right)$ $>0)$. In order for the terms of the series in $(1.2 \mathrm{a}),(1.2 \mathrm{~b})$ to be finite, the variable $z$ should be chosen such that it is nonzero (modulo $\frac{\pi}{i \log q}$ ) and such that $g_{r} \pm z$ is not a negative integer (modulo $\frac{2 \pi}{i \log q}$ ). The identities in (1.1a) and (1.1b) pass over into the Bailey and Dougall sums in (1.2a), (1.2b) after division by the middle term corresponding to $\lambda=0$ (which is nonzero with these restrictions on $z$ ) and rewriting of the resulting 1.h.s. with the aid of the relations 
$(a ; q)_{\infty} /\left(a q^{\lambda} ; q\right)_{\infty}=(a ; q)_{\lambda}$ and $\Gamma(a+\lambda) / \Gamma(a)=(a)_{\lambda}$ as well as the reflection properties $(a ; q)_{\lambda}\left(a^{-1} q ; q\right)_{-\lambda}=(-a)^{\lambda} q^{\lambda(\lambda-1) / 2}$ and $(a)_{\lambda}(1-a)_{-\lambda}=(-1)^{\lambda}$, respectively.

The plan of the paper reads as follows. In Section 2 a multidimensional version of the sums in (1.1a) and (1.1b) is discussed (Theorem 1). For $0<q<1$ the sum under consideration amounts to a generalization corresponding to the case of a nonreduced root system of summation formulas appearing in recent work of Macdonald [Ma2] associated to the (affine) reduced root systems. Alternative representations lead to certain multidimensional analogues of the Bailey ${ }_{6} \psi_{6}$ sum (1.2a) and the Dougall ${ }_{5} H_{5}$ sum (1.2b), as well as to multiple summation formulas of the type studied by Aomoto and Ito [Ao, I]. Section 3 describes the specialization to nonterminating and terminating multidimensional versions of Rogers' very-well-poised ${ }_{6} \phi_{5}$ sum [Ro, GR] and Dougall's very-well-poised ${ }_{5} F_{4}$ sum [Do, GR] (Theorem 2 and 3). The resulting terminating sums (of Theorem 3) may be used to arrive at product formulas for the norms of recently introduced $(q$-)Racah polynomials in several variables [DS] that generalize the well-known one-variable $(q-)$ Racah polynomials of Askey and Wilson [AW, W, GR]. The technicalities pertaining to the proof for the multiple bilateral summation formulas of Section 2 are relegated to Section 4 and an appendix at the end of the paper (in which the convergence of the series is demonstrated). The proof in Section 4 is based on a recurrence relation for the generalized Macdonald type sum (of Theorem 1), which is derived using a technique very similar to that employed by Gustafson in his proof of the Selberg type multivariable Askey-Wilson integral studied in [Gu3].

The sums considered in this paper are not the only/first possible (nontrivial) multidimensional generalizations of the ${ }_{5} F_{4},{ }_{6} \phi_{5},{ }_{5} H_{5}$ and ${ }_{6} \psi_{6}$ summation formulas. An important class of very-well-poised (basic) hypergeometric summation formulas associated with the (special) unitary group $(S) U(n)$ (type $A$ root system) can e.g. be found in the works of Holman [Ho] $\left({ }_{5} F_{4}\right.$ type), Milne [M1, M2] ( ${ }_{6} \phi_{5}$ type) and Gustafson [Gu1] $\left({ }_{5} H_{5}\right.$ and $\left.{ }_{6} \psi_{6}{ }^{7} 7 \mathrm{pe}\right)$. Gustafson moreover generalized his $(S) U(n)$ type multiple ${ }_{5} H_{5}$ and ${ }_{6} \psi_{6}$ sums of [Gu1] to the case of an arbitrary classical simple Lie group [Gu2]. For the symplectic group $S p(n)$ (type $C$ root system) truncated versions of Gustafson's ${ }_{6} \psi_{6}$ sum giving rise to terminating and nonterminating multiple ${ }_{6} \phi_{5}$ summation formulas were discussed by Lilly and Milne [LM]. Very recently, still other multidimensional versions of the ${ }_{6} \psi_{6}$ and ${ }_{6} \phi_{5}$ summation formulas associated with the type $C$ root system were presented by Schlosser [Sch]. 
All these multidimensional generalizations of the ${ }_{5} F_{4},{ }_{6} \phi_{5},{ }_{5} H_{5}$ and ${ }_{6} \psi_{6}$ summation formulas occurring in the literature are of a different type than those studied below. We will, however, have the opportunity in Section 4 to employ Gustafson's multiple ${ }_{5} H_{5}$ and ${ }_{6} \psi_{6}$ summation formulas from [Gu2] for the symplectic group $S p(n)$ (type $C$ root system), when deriving the recurrence relation that leads to the proof of the Macdonald type sum given by Theorem 1.

Remark. We have adopted the following (standard) conventions regarding the notation of $q$-shifted factorials and Pochhammer symbols, respectively [GR]

(1.3a) $(a ; q)_{m} \equiv \begin{cases}1 & \text { for } m=0 \\ (1-a)(1-a q) \cdots\left(1-a q^{m-1}\right) & \text { for } m=1,2,3, \cdots \\ \frac{1}{\left(1-a q^{-1}\right)\left(1-a q^{-2}\right) \cdots\left(1-a q^{m}\right)} & \text { for } m=-1,-2,-3, \cdots\end{cases}$

and

$$
(a)_{m} \equiv \begin{cases}1 & \text { for } m=0 \\ a(a+1) \cdots(a+m-1) & \text { for } m=1,2,3, \cdots \\ \frac{1}{(a-1)(a-2) \cdots(a+m)} & \text { for } m=-1,-2,-3, \cdots\end{cases}
$$

(where for negative $m$ it is assumed that the value of $a$ is such that the denominator does not vanish). One has

$$
(a ; q)_{m}=\frac{(a ; q)_{\infty}}{\left(a q^{m} ; q\right)_{\infty}} \quad \text { and } \quad(a)_{m}=\frac{\Gamma(a+m)}{\Gamma(a)}
$$

where $\Gamma(\cdot)$ represents the gamma function and

$$
(a ; q)_{\infty} \equiv \prod_{k=0}^{\infty}\left(1-a q^{k}\right)
$$

(here it is assumed that $|q|<1$ ). We furthermore use the abbreviation

$$
\begin{gathered}
\left(a_{1}, \cdots, a_{p} ; q\right)_{m} \equiv\left(a_{1} ; q\right)_{m} \cdots\left(a_{p} ; q\right)_{m}, \\
\left(a_{1}, \cdots, a_{p}\right)_{m} \equiv\left(a_{1}\right)_{m} \cdots\left(a_{p}\right)_{m} .
\end{gathered}
$$


One-variable bilateral (basic) hypergeometric series and their unilateral counterparts are denoted by

$$
\begin{gathered}
{ }_{p} \psi_{p}\left(\begin{array}{l}
a_{1}, \cdots, a_{p} \\
b_{1}, \cdots, b_{p}
\end{array} ;, \zeta\right) \equiv \sum_{\lambda \in Z} \frac{\left(a_{1}, \cdots, a_{p} ; q\right)_{\lambda}}{\left(b_{1}, \cdots, b_{p} ; q\right)_{\lambda}} \zeta^{\lambda}, \\
{ }_{p} H_{p}\left(\begin{array}{l}
a_{1}, \cdots, a_{p} \\
b_{1}, \cdots, b_{p}
\end{array} \zeta\right) \equiv \sum_{\lambda \in Z} \frac{\left(a_{1}, \cdots, a_{p}\right)_{\lambda}}{\left(b_{1}, \cdots, b_{p}\right)_{\lambda}} \zeta^{\lambda}
\end{gathered}
$$

and

$$
\begin{gathered}
{ }_{p} \phi_{p-1}\left(\begin{array}{l}
a_{1}, \cdots, a_{p} \\
b_{1}, \cdots, b_{p-1}
\end{array} ; q, \zeta\right) \equiv \sum_{\lambda \in N} \frac{\left(a_{1}, \cdots, a_{p} ; q\right)_{\lambda}}{\left(b_{1}, \cdots, b_{p-1}, q ; q\right)_{\lambda}} \zeta^{\lambda}, \\
{ }_{p} F_{p-1}\left(\begin{array}{l}
a_{1}, \cdots, a_{p} \\
b_{1}, \cdots, b_{p-1}
\end{array} ; \zeta\right) \equiv \sum_{\lambda \in N} \frac{\left(a_{1}, \cdots, a_{p}\right)_{\lambda}}{\left(b_{1}, \cdots, b_{p-1}, 1, q\right)_{\lambda}} \zeta^{\lambda}
\end{gathered}
$$

(where $N$ includes the number zero).

\section{§2. Multiple Analogues of Very-Well-Poised Bilateral (Basic) Hypergeometric Series}

Note. In this section it is always assumed that the nome $q$ lies in the open interval $] 0,1[$ (corresponding to the case of basic hypergeometric series) or that it degenerates to $q=1$ (corresponding to the case of ordinary hypergeometric series).

\subsection{Notation}

We will first set up some notational preliminaries. The reader may wish to skip this part at first reading and refer back to it when needed.

To describe the multiple summation formulas below it is convenient to introduce the functions $\mathscr{C}_{+, q}(\mathbf{x}), \mathscr{C}_{-, q}(\mathbf{x}), \hat{\mathscr{C}}_{+, q}(\mathbf{x})$ and $\hat{\mathscr{C}}_{-, q}(\mathbf{x})$ given for $0<q<1$ by

$$
\begin{aligned}
\mathscr{C}_{+, q}(\mathbf{x})= & \prod_{1 \leq j<k \leq n} \frac{\left(q^{1+x_{j}+x_{k}}, q^{1+x_{j}-x_{k}} ; q\right)_{\infty}}{\left(q^{1+g+x_{j}+x_{k}}, q^{1+g+x_{j}-x_{k}} ; q\right)_{\infty}} \\
& \times \prod_{1 \leq j \leq n} \frac{\left(q^{1+2 x_{j}} ; q\right)_{\infty}}{\prod_{r=1}^{4}\left(q^{1+g_{r}+x_{j}} ; q\right)_{\infty}}
\end{aligned}
$$

$$
\mathscr{C}_{-. q}(\mathbf{x})=q^{-\Sigma_{j=1}^{n}\left(1+2 \hat{\rho}_{j}\right) x_{j}} \prod_{1 \leq j<k \leq n} \frac{\left(q^{-g+x_{j}+x_{k}}, q^{-g+x_{j}-x_{k}} ; q\right)_{\infty}}{\left(q^{x_{j}+x_{k}}, q^{x_{j}-x_{k}} ; q\right)_{\infty}}
$$




$$
\times \prod_{1 \leq j \leq n} \frac{\Pi_{r=1}^{4}\left(q^{-g_{r}+x_{j}} ; q\right)_{\infty}}{\left(q^{2 x_{j}} ; q\right)_{\infty}}
$$

(2.1c) $\hat{\mathscr{C}}_{+. q}(\mathbf{x})=\prod_{1 \leq j<k \leq n} \frac{\left(q^{1+x_{j}+x_{k}}, q^{1+x_{j}-x_{k}} ; q\right)_{\infty}}{\left(q^{1+g+x_{j}+x_{k}}, q^{1+g+x_{j}-x_{k}} ; q\right)_{\infty}}$

$$
\times \prod_{1 \leq j \leq n} \frac{\left(q^{1+2 x_{j}} ; q\right)_{\infty}}{\Pi_{r=1}^{4}\left(q^{1+\hat{g}_{r}+x_{j}} ; q\right)_{\infty}}
$$

(2.1d) $\quad \hat{\mathscr{C}}_{-, q}(\mathbf{x})=\prod_{1 \leq j<k \leq n} \frac{\left(q^{1-g+x_{j}+x_{k}}, q^{1-g+x_{j}-x_{k}} ; q\right)_{\infty}}{\left(q^{1+x_{j}+x_{k}}, q^{1+x_{j}-x_{k}} ; q\right)_{\infty}}$

$$
\times \prod_{1 \leq j \leq n} \frac{\Pi_{r=1}^{4}\left(q^{1-\hat{g}_{r}+x_{j}} ; q\right)_{\infty}}{\left(q^{1+2 x_{j}} ; q\right)_{\infty}}
$$

and for the degenerate case $q=1$ by

$$
\begin{aligned}
\mathscr{C}_{+.1}(\mathbf{x})= & \prod_{1 \leq j<k \leq n} \frac{\Gamma\left(1+g+x_{j}+x_{k}\right) \Gamma\left(1+g+x_{j}-x_{k}\right)}{\Gamma\left(1+x_{j}+x_{k}\right) \Gamma\left(1+x_{j}-x_{k}\right)} \\
& \times \prod_{1 \leq j \leq n} \frac{\Pi_{r=1}^{4} \Gamma\left(1+g_{r}+x_{j}\right)}{\Gamma\left(1+2 x_{j}\right)}
\end{aligned}
$$

$$
\begin{aligned}
\mathscr{C}_{-, 1}(\mathbf{x})= & \prod_{1 \leq j<k \leq n} \frac{\Gamma\left(x_{j}+x_{k}\right) \Gamma\left(x_{j}-x_{k}\right)}{\Gamma\left(-g+x_{j}+x_{k}\right) \Gamma\left(-g+x_{j}-x_{k}\right)} \\
& \times \prod_{1 \leq j \leq n} \frac{\Gamma\left(2 x_{j}\right)}{\Pi_{r=1}^{4} \Gamma\left(-g_{r}+x_{j}\right)}
\end{aligned}
$$

$$
\begin{aligned}
\hat{\mathscr{C}}_{+.1}(\mathbf{x})= & \prod_{1 \leq j<k \leq n} \frac{\Gamma\left(1+g+x_{j}+x_{k}\right) \Gamma\left(1+g+x_{j}-x_{k}\right)}{\Gamma\left(1+x_{j}+x_{k}\right) \Gamma\left(1+x_{j}-x_{k}\right)} \\
& \times \prod_{1 \leq j \leq n} \frac{\Pi_{r=1}^{4} \Gamma\left(1+\hat{g}_{r}+x_{j}\right)}{\Gamma\left(1+2 x_{j}\right)},
\end{aligned}
$$

$$
\begin{aligned}
\hat{\mathscr{C}}_{-, 1}(\mathbf{x})= & \prod_{1 \leq j<k \leq n} \frac{\Gamma\left(1+x_{j}+x_{k}\right) \Gamma\left(1+x_{j}-x_{k}\right)}{\Gamma\left(1-g+x_{j}+x_{k}\right) \Gamma\left(1-g+x_{j}-x_{k}\right)} \\
& \times \prod_{1 \leq j \leq n} \frac{\Gamma\left(1+2 x_{j}\right)}{\Pi_{r=1}^{4} \Gamma\left(1-\hat{g}_{r}+x_{j}\right)}
\end{aligned}
$$

The parameters $\hat{g}_{r}, r=1,2,3,4$, are related to the parameters $g_{r}, r=1,2,3$, 
4 , by the transformation

$$
\left(\begin{array}{l}
\hat{g}_{a} \\
\hat{g}_{b} \\
\hat{g}_{c} \\
\hat{g}_{d}
\end{array}\right)=\frac{1}{2}\left(\begin{array}{cccc}
1 & 1 & 1 & 1 \\
1 & 1 & -1 & -1 \\
1 & -1 & 1 & -1 \\
1 & -1 & -1 & 1
\end{array}\right)\left(\begin{array}{l}
g_{a} \\
g_{b} \\
g_{c} \\
g_{d}
\end{array}\right)
$$

where $a, b, c$ and $d$ denote a (fixed but otherwise arbitrary) permutation of the indices $1,2,3$ and 4 (i.e., one has that $\{a, b, c, d\}=\{1,2,3,4\}$ ). We may in fact set $a=1, b=2, c=3$ and $d=4$ without loss of generality, but here we have preferred not to fix such choice explicitly in order to reflect in our notation the invariance of the construction with respect to permutations of the parameters $g_{1}, g_{2}, g_{3}, g_{4}$.

We will furthermore employ the vectors $\rho$ and $\hat{\rho}$ with components given by

$$
\rho_{j}=(n-j) g+g_{a} \quad \text { and } \quad \hat{\rho}_{j}=(n-j) g+\hat{g}_{a}
$$

$(j=1, \cdots, n)$ and the Jacobi theta function

$$
\theta(\zeta)=\sum_{m=-\infty}^{\infty}(-1)^{m} q^{m(m-1) / 2 \zeta^{m}}
$$

This theta function satisfies the quasi-periodicity relation

$$
\theta(q \zeta)=-\zeta^{-1} \theta(\zeta)
$$

and admits the product representation

$$
\theta(\zeta)=\left(q, \zeta, q \zeta^{-1} ; q\right)_{\infty} .
$$

The equality of the r.h.s. of (2.5) and (2.7) hinges on a classic bilateral summation formula known as the Jacobi triple product identity (see e.g. [GR]). The $q=1$ counterpart of $\theta\left(q^{z}\right)$ is given by the sine function $\sin (\pi z)$ and the analogue of the product formula boils in this degenerate situation down to the reflection relation for the gamma function

$$
\sin (\pi z)=\frac{\pi}{\Gamma(z) \Gamma(1-z)}
$$

The corresponding period lattice $\Omega_{q} \subset C$ is given by 


$$
\Omega_{q}=\left\{\begin{array}{lll}
\mathbb{Z}+\frac{2 \pi}{i \log (q)} & \text { for } & 0<q<1 \\
\mathbb{Z} & \text { for } & q=1 .
\end{array}\right.
$$

\subsection{A Macdonald Type Sum}

The following theorem describes a multidimensional generalization of the summation formulas (1.1a) (when $0<q<1$ ) and (1.1b) (when $q=1$ ) and reduces to these formulas for $n=1$.

Theorem $\mathbb{1}$. Let $0<q \leq 1$. For parameters subject to the condition

$$
\operatorname{Re}\left(1+2(n-j) g+g_{1}+g_{2}+g_{3}+g_{4}\right)>0
$$

(with $j=1, \cdots, n$ ), one has that

$$
\sum_{\lambda \in \mathbb{Z}^{n}} \frac{1}{\mathscr{C}_{+, q}(z+\lambda) \mathscr{C}_{+, q}(-z-\lambda)}=\frac{\hat{\mathscr{C}}_{-, q}(\hat{\rho})}{\hat{\mathscr{C}}_{+, q}(\hat{\rho})}
$$

and the series on the l.h.s. converges in absolute value. Here it is assumed that $z \in \mathbb{C}^{n}$ with the combinations $z_{j}+z_{k}, z_{j}-z_{k}(1 \leq j<k \leq n)$ and $2 z_{j}(1 \leq j \leq n)$ being nonzero modulo the lattice $\Omega_{q}(2.9)$.

The genericity condition on the components of the vector $z$ ensures that the denominators of the terms on the 1.h.s. do not vanish; the proof that the series under consideration converges in absolute value can be found in the appendix at the end of the paper. The evaluation of the sum hinges on a recurrence relation that is derived by means of a technique due to Gustafson [Gu3], who used it to evaluate a Selberg type multivariable generalization of the Askey-Wilson integral (see [GR] and references therein for a discussion of the Askey-Wilson integral). The details of this derivation, leading to a proof for the fact that the value of the sum is given by the $z$-independent constant on the r.h.s. of (2.11), are relegated to Section 4 .

A basic hypergeometric summation formula closely related to that described by the theorem has been derived recently by Macdonald using the properties of affine Weyl groups and root systems [Ma2]. More specifically, the sum considered by Macdonald is associated to an arbitrary reduced root system and Theorem 1 (with $0<q<1$ ) may be viewed as the extension to the case of a nonreduced root system. For special values of the parameters $g_{1}, g_{2}, g_{3}, g_{4}$, the basic hypergeometric sum in (2.11) reduces to the Macdonald sums related 
to the reduced root systems of classical type corresponding to the $B, C$ and $D$ series.

The evaluation constant on the r.h.s. of (2.11) given by

$$
\begin{aligned}
\frac{\hat{\mathscr{C}}_{-, q}(\hat{\rho})}{\hat{\mathscr{C}}_{+, q}(\hat{\rho})}=\prod_{1 \leq j<k \leq n} \frac{\left(q^{1+g+\hat{\rho}_{j}+\hat{\rho}_{k}}, q^{1+g+\hat{\rho}_{j}-\hat{\rho}_{k}} ; q\right)_{\infty}}{\left(q^{1+\hat{\rho}_{j}+\hat{\rho}_{k}}, q^{1+\hat{\rho}_{j}-\hat{\rho}_{k}} ; q\right)_{\infty}} \\
\times \frac{\left(q^{1-g+\hat{\rho}_{j}+\hat{\rho}_{k}}, q^{1-g+\hat{\rho}_{J}-\hat{\rho}_{k}} ; q\right)_{\infty}}{\left(q^{1+\hat{\rho}_{j}+\hat{\rho}_{k}}, q^{1+\hat{\rho}_{j}-\hat{\rho}_{k}} ; q\right)_{\infty}} \\
\times \prod_{1 \leq j \leq n} \frac{\Pi_{r=1}^{4}\left(q^{1+\hat{g}_{r}+\hat{\rho}_{J}}, q^{1-\hat{g}_{r}+\hat{\rho}_{J}} ; q\right)_{\infty}}{\left(q^{1+2 \hat{\rho}_{j}} ; q\right)_{\infty}^{2}}
\end{aligned}
$$

for $0<q<1$ and by

$$
\begin{aligned}
\frac{\hat{\mathscr{C}}_{-, 1}(\hat{\rho})}{\hat{\mathscr{C}}_{+, 1}(\hat{\rho})}=\prod_{1 \leq j<k \leq n} \frac{\Gamma\left(1+\hat{\rho}_{j}+\hat{\rho}_{k}\right) \Gamma\left(1+\hat{\rho}_{j}-\hat{\rho}_{k}\right)}{\Gamma\left(1+g+\hat{\rho}_{j}+\hat{\rho}_{k}\right) \Gamma\left(1+g+\hat{\rho}_{j}-\hat{\rho}_{k}\right)} \\
\times \frac{\Gamma\left(1+\hat{\rho}_{j}+\hat{\rho}_{k}\right) \Gamma\left(1+\hat{\rho}_{j}-\hat{\rho}_{k}\right)}{\Gamma\left(1-g+\hat{\rho}_{j}+\hat{\rho}_{k}\right) \Gamma\left(1-g+\hat{\rho}_{j}-\hat{\rho}_{k}\right)} \\
\times \prod_{1 \leq j \leq n} \frac{\Gamma\left(1+2 \hat{\rho}_{j}\right)^{2}}{\Pi_{r=1}^{4} \Gamma\left(1+\hat{g}_{r}+\hat{\rho}_{j}\right) \Gamma\left(1-\hat{g}_{r}+\hat{\rho}_{j}\right)}
\end{aligned}
$$

for $q=1$, may be rewritten by canceling common factors in the numerator and the denominator as

$$
\begin{aligned}
& \frac{\hat{\mathscr{C}}_{-, q}(\hat{\rho})}{\hat{\mathscr{C}}_{+, q}(\hat{\rho})}= \\
& \begin{cases}\prod_{1 \leq j \leq n} \frac{\left(q, q^{1+j g} ; q\right)_{\infty} \Pi_{1 \leq r<s \leq 4}\left(q^{1+(n-j) g+g_{r}+g_{s}} ; q\right)_{\infty}}{\left(q^{1+g}, q^{1+(2 n-j-1) g+g_{1}+g_{2}+g_{3}+g_{4}} ; q\right)_{\infty}} & \text { for } \quad 0<q<1 \\
\prod_{1 \leq j \leq n} \frac{\Gamma(1+g) \Gamma\left(1+(2 n-j-1) g+g_{1}+g_{2}+g_{3}+g_{4}\right)}{\Gamma(1+j g) \Pi_{1 \leq r<s \leq 4} \Gamma\left(1+(n-j) g+g_{r}+g_{s}\right)} & \text { for } \quad q=1 .\end{cases}
\end{aligned}
$$

It is clear from this last formula and the explicit expressions for $\mathscr{C}_{+, q}(\mathbf{x})$ in (2.1a), (2.2a) that the summation formula of Theorem 1 reduces to the sums in (1.1a), (1.1b) for $n=1$.

\subsection{A Generalized Aomoto-Ito Sum}

In $[\mathrm{Ao}, \mathrm{I}]$ Aomoto and Ito presented an evaluation conjecture for certain 
$q$-Selberg type Jackson integrals (sums) associated to the reduced root systems. For the type $A$ root system, the validity of the summation formula in question was inferred by Aomoto (in [Ao]) and also by Kaneko [Ka]. In this special case the sum amounts to an extension of a $q$-Selberg Jackson integral (sum) due to Askey, Kadell, Habsieger and Evans $[A, K, H, E]$. For the remaining root systems, the validity of the Aomoto-Ito formula was verified in the rank two case by Ito, who also extended the result to the case of a rank two nonreduced $B C$ type root system [I]. Recently, Macdonald observed that the Aomoto-Ito formula follows for arbitrary reduced root system from what we have dubbed here 'the Macdonald sum' (i.e., the analogue of the sum in (2.11) for a reduced root system) [Ma2]. The main purpose of the present section is to derive a generalized Aomoto-Ito type sum for the nonreduced (affine) root systems. To this end, we will rewrite the sum of Theorem 1 following (in essence) Macdonald's treatment for the case of a reduced root system.

The main point of our discussion is that multiplication of both sides of (2.11) by the factor $\mathscr{C}_{+, q}(-z) / \mathscr{C}_{-, q}(z)$ leads to

A generalized Aomoto-Ito sum

$$
\sum_{\lambda \in \mathbb{Z}^{n}} \frac{1}{\mathscr{C}_{+. q}(z+\lambda) \mathscr{C}_{-, q}(z+\lambda)}=\frac{\mathscr{C}_{+. q}(-z)}{\mathscr{C}_{-. q}(z)} \frac{\hat{\mathscr{C}}_{-, q}(\hat{\rho})}{\hat{\mathscr{C}}_{+, q}(\hat{\rho})},
$$

where it is again assumed that the parameters $g, g_{r}(r=1,2,3,4)$ satisfy the convergence condition in (2.10). To avoid poles one should choose $z$ such that $-g+z_{j} \pm z_{k}(1 \leq j<k \leq n)$ and $-g_{r}+z_{j}(r=1,2,3,4 ; 1 \leq j \leq n)$ are nonzero modulo the lattice $\Omega_{q}$ (2.9) (cf. the expressions below). In fact, the identity (2.14) may be viewed as an equality between meromorphic functions of $z$. More specifically, the 1.h.s. and r.h.s. of (2.14) are equal as a meromorphic function in $z_{j}$ (where $j$ is arbitrary but fixed) with poles congruent (modulo $\Omega_{q}$ (2.9)) to $z_{j}= \pm\left(g-z_{k}\right)(k<j), z_{j}=g \pm z_{k}(k>j)$ and $z_{j}=g_{r}(r=1,2,3,4)$.

In order to arrive at the Aomoto-Ito type sum (2.14) from (2.11), we have used for the 1.h.s. the fact that the multiplier $\mathscr{C}_{+, q}(-z) / \mathscr{C}_{-, q}(z)$ is periodic in $z$

$$
\frac{\mathscr{C}_{+, q}(-z)}{\mathscr{C}_{-, q}(z)}=\frac{\mathscr{C}_{+, q}(-z-\lambda)}{\mathscr{C}_{-, q}(z+\lambda)}, \quad \text { for } \quad \lambda \in \mathbb{Z}^{n} .
$$

This periodicity is not difficult to see by inferring that the factor $\mathscr{C}_{+, q}(-z) / \mathscr{C}_{-, q}(z)$ is invariant with respect to translations in $z$ over the unit vectors $e_{j}, j=1, \cdots, n$ 
(of the standard basis in $\boldsymbol{R}^{n}$ ) using the elementary shift properties for the $q$-shifted factorial (viz. $(q a ; q)_{\infty}=(a ; q)_{\infty} /(1-a)$ ) and gamma function (viz. $\Gamma(a+1)=a \Gamma(a)$ ), respectively. (For a still simpler way to deduce the periodicity see below.) For $0<q<1$ and special values of the parameters $g_{1}, g_{2}, g_{3}, g_{4}$, the summation formula in (2.14) amounts to the $B C$ type Aomoto-Ito sum (see the appendix of [I]). By further specialization of the parameters one recovers the Aomoto-Ito sums associated to the reduced root systems of type $B, C$ and $D$.

To make the contact with $[\mathrm{Ao}, \mathrm{I}]$ and [Ma2] more explicit, it is helpful to observe that for $0<q<1$ the terms in (2.14) may be written as

$$
\begin{aligned}
& \frac{1}{\mathscr{C}_{+, q}(\mathbf{x}) \mathscr{C}_{-, q}(\mathbf{x})}=q^{\Sigma_{j=1}^{n}\left(1+2 \hat{\rho}_{j}\right) x_{j}} \\
& \times \prod_{1 \leq j<k \leq n}\left(1-q^{x_{j}+x_{k}}\right)\left(1-q^{x_{j}-x_{k}}\right) \frac{\left(q^{1+g+x_{j}+x_{k}}, q^{1+g+x_{j}-x_{k}} ; q\right)_{\infty}}{\left(q^{-g+x_{j}+x_{k}}, q^{-g+x_{j}-x_{k}} ; q\right)_{\infty}} \\
& \times \prod_{1 \leq j \leq n}\left(1-q^{2 x_{j}}\right) \frac{\left(q^{1+g_{1}+x_{j}}, q^{1+g_{2}+x_{j}}, q^{1+g_{3}+x_{j}}, q^{1+g_{4}+x_{j}} ; q\right)_{\infty}}{\left(q^{-g_{1}+x_{j}}, q^{-g_{2}+x_{j}}, q^{-g_{3}+x_{j}}, q^{-g_{4}+x_{j}} ; q\right)_{\infty}}
\end{aligned}
$$

(with $\mathbf{x}=\boldsymbol{z}+\lambda$ ). Furthermore, the periodic Aomoto factor $\mathscr{C}_{+, q}(-\boldsymbol{z}) / \mathscr{C}_{-, q}(\boldsymbol{z})$ on the r.h.s. is conveniently rewritten in terms of the Jacobi theta function $\theta(2.5)$ as follows

$$
\begin{aligned}
& \frac{\mathscr{C}_{+, q}(-z)}{\mathscr{C}_{-, q}(z)}=q^{\Sigma_{j=1}^{n}\left(1+2 \hat{\rho}_{j}\right) z_{j}} \\
& \times \prod_{1 \leq j<k \leq n} \frac{\theta\left(q^{z_{j}+z_{k}}\right) \theta\left(q^{z_{j}-z_{k}}\right)}{\theta\left(q^{-g+z_{j}+z_{k}}\right) \theta\left(q^{-g+z_{j}-z_{k}}\right)} \prod_{1 \leq j \leq n} \frac{(q ; q)_{\infty}^{3} \theta\left(q^{2 z_{j}}\right)}{\prod_{1 \leq r \leq 4} \theta\left(q^{-g_{r}+z_{j}}\right)}
\end{aligned}
$$

(this is clear from the product representation for $\theta(\zeta)$ in (2.7)). Observe that the periodicity relation (2.15) is now easily deduced using the quasi-periodicity relation (2.6) for the theta function.

For $q=1$ the summation formula in (2.14) constitutes a degeneration of the (generalized) Aomoto-Ito formula. The summand on the 1.h.s. is in this case governed by

$$
\frac{1}{\mathscr{C}_{+, 1}(\mathbf{x}) \mathscr{C}_{-, 1}(\mathbf{x})}
$$




$$
\begin{aligned}
= & \prod_{1 \leq j<k \leq n} \frac{\left(x_{j}+x_{k}\right)\left(x_{j}-x_{k}\right) \Gamma\left(-g+x_{j}+x_{k}\right) \Gamma\left(-g+x_{j}-x_{k}\right)}{\Gamma\left(1+g+x_{j}+x_{k}\right) \Gamma\left(1+g+x_{j}-x_{k}\right)} \\
& \times \prod_{1 \leq j \leq n} \frac{2 x_{j} \Gamma\left(-g_{1}+x_{j}\right) \Gamma\left(-g_{3}+x_{j}\right) \Gamma\left(-g_{3}+x_{j}\right) \Gamma\left(-g_{4}+x_{j}\right)}{\Gamma\left(1+g_{1}+x_{j}\right) \Gamma\left(1+g_{2}+x_{j}\right) \Gamma\left(1+g_{3}+x_{j}\right) \Gamma\left(1+g_{4}+x_{j}\right)}
\end{aligned}
$$

$(\mathbf{x}=z+\lambda)$ and the degenerate Aomoto factor on the r.h.s. can now be written in the manifestly periodic form

$$
\begin{aligned}
\frac{\mathscr{C}_{+, 1}(-z)}{\mathscr{C}_{-.1}(z)}= & \prod_{1 \leq j<k \leq n} \frac{\sin \pi\left(z_{j}+z_{k}\right) \sin \pi\left(z_{j}-z_{k}\right)}{\sin \pi\left(-g+z_{j}+z_{k}\right) \sin \pi\left(-g+z_{j}-z_{k}\right)} \\
& \times \prod_{1 \leq j \leq n} \frac{\pi^{3} \sin \left(2 \pi z_{j}\right)}{\Pi_{1 \leq r \leq 4} \sin \pi\left(-g_{r}+z_{j}\right)}
\end{aligned}
$$

(where we have used the reflection equation (2.8)).

For $n=1$ the generalized Aomoto-Ito summation formula reduces (after division by a common factor $\left.q^{\left(1+g_{1}+g_{2}+g_{3}+g_{4}\right) z}\right)$ to

$$
\begin{aligned}
\sum_{\lambda \in \mathbf{Z}} q^{\left(1+g_{1}+g_{2}+g_{3}+g_{4}\right) \lambda}\left(1-q^{2 z+2 \lambda}\right) \prod_{1 \leq r \leq 4} \frac{\left(q^{1+g_{r}+z+\lambda} ; q\right)_{\infty}}{\left(q^{-g_{r}+z+\lambda} ; q\right)_{\infty}} \\
=\frac{(q ; q)_{\infty}^{3} \theta\left(q^{2 z}\right)}{\Pi_{1 \leq r \leq 4} \theta\left(q^{-g_{r}+z}\right)} \frac{(q ; q)_{\infty} \Pi_{1 \leq r<s \leq 4}\left(q^{1+g_{r}+g_{s}} ; q\right)_{\infty}}{\left(q^{1+g_{1}+g_{2}+g_{3}+g_{4}} ; q\right)_{\infty}} .
\end{aligned}
$$

and the corresponding $q=1$ degeneration reads

$$
\begin{aligned}
\sum_{\lambda \in \mathbf{Z}}\left((2 z+2 \lambda) \prod_{1 \leq r \leq 4} \frac{\Gamma\left(-g_{r}+z+\lambda\right)}{\Gamma\left(1+g_{r}+z+\lambda\right)}\right) \\
=\frac{\pi^{3} \sin \left(2 \pi z_{j}\right)}{\Pi_{1 \leq r \leq 4} \sin \pi\left(-g_{r}+z_{j}\right)} \frac{\Gamma\left(1+g_{1}+g_{2}+g_{3}+g_{4}\right)}{\Pi_{1 \leq r<s \leq 4} \Gamma\left(1+g_{r}+g_{s}\right)}
\end{aligned}
$$

\subsection{A Multiple Bailey ${ }_{6} \psi_{6}$ and Dougall ${ }_{5} H_{5}$ Sum}

Division of the Macdonald type sum (2.11) by the middle term $1 /\left(\mathscr{C}_{+, q}(z) \mathscr{C}_{+, q}(-z)\right)$ (corresponding to $\lambda=0$ ), or equivalently, division of the Aomoto-Ito type sum $(2.14)$ by the middle term $1 /\left(\mathscr{C}_{+, q}(z) \mathscr{C}_{-. q}(z)\right)$ leads us to

A multiple Bailey/Dougall sum

$$
\sum_{\lambda \in \mathbb{Z}^{n}} \frac{\mathscr{C}_{+, q}(z) \mathscr{C}_{+, q}(-z)}{\mathscr{C}_{+, q}(z+\lambda) \mathscr{C}_{+, q}(-z-\lambda)}=\mathscr{C}_{+, q}(z) \mathscr{C}_{+, q}(-z) \frac{\hat{\mathscr{C}}_{-, q}(\hat{\rho})}{\hat{\mathscr{C}}_{+, q}(\hat{\rho})}
$$


or equivalently

$$
\sum_{\lambda \in \mathbf{Z}^{n}} \frac{\mathscr{C}_{+. q}(z) \mathscr{C}_{-, q}(-z)}{\mathscr{C}_{+, q}(z+\lambda) \mathscr{C}_{-, q}(z+\lambda)}=\mathscr{C}_{+, q}(z) \mathscr{C}_{+, q}(-z) \frac{\hat{\mathscr{C}}_{-. q}(\hat{\rho})}{\hat{\mathscr{C}}_{+, q}(\hat{\rho})}
$$

(the equality of the terms on the 1.h.s. of (2.22a) and (2.22b) traces back to the periodicity relation (2.15)), where the parameters $g, g_{r}$ again satisfy the convergence condition in (2.10). To avoid poles, the components of $z$ should now be chosen subject to the genericity condition that $2 z_{j}, z_{j} \pm z_{k}(j \neq k)$ is nonzero (modulo $\frac{2 \pi}{i \log q}$ ) and that $g_{r} \pm z_{j}, g \pm z_{j} \pm z_{k}(j \neq k)$ is not a negative integer (modulo $\frac{2 \pi}{i \log q}$ ). In order to facilitate the comparison with the classical one-variable Bailey and Dougall sums in (1.2a) and (1.2b), it is useful to display the terms on the 1.h.s. of the multiple summation formula $(2.22 \mathrm{a}),(2.22 \mathrm{~b})$ somewhat more explicitly. One has for $0<q<1$ that

$$
\begin{gathered}
\frac{\mathscr{C}_{+. q}(z) \mathscr{C}_{-, q}(z)}{\mathscr{C}_{+, q}(z+\lambda) \mathscr{C}_{-, q}(z+\lambda)}=q^{\Sigma_{J=1}^{n}\left(1+2 \hat{\rho}_{j}\right) \lambda_{j}} \\
\times \prod_{1 \leq j<k \leq n}\left(\frac{1-q^{z_{j}+z_{k}+\lambda_{j}+\lambda_{k}}}{1-q^{z_{j}+z_{k}}} \frac{1-q^{z_{J}-z_{k}+\lambda_{j}-\lambda_{k}}}{1-q^{z_{j}-z_{k}}}\right) \\
\times \frac{\left(q^{-g+z_{j}+z_{k}} ; q\right)_{\lambda_{j}+\lambda_{k}}\left(q^{-g+z_{j}-z_{k}} ; q\right)_{\lambda_{j}-\lambda_{k}}}{\left(q^{1+g+z_{j}+z_{k}} ; q\right)_{\lambda_{j}+\lambda_{k}}\left(q^{1+g+z_{j}-z_{k}} ; q\right)_{\lambda_{j}-\lambda_{k}}} \\
\times \prod_{1 \leq j \leq n}\left(\frac{1-q^{2 z_{j}+2 \lambda_{j}}}{1-q^{2 z_{j}}}\right) \frac{\left(q^{-g_{1}+z_{j}}, q^{-g_{2}+z_{j}}, q^{-g_{3}+z_{j}}, q^{-g_{4}+z_{j}} ; q\right)_{\lambda_{j}}}{\left(q^{1+g_{1}+z_{j}}, q^{1+g_{2}+z_{j}}, q^{1+g_{3}+z_{j}}, q^{1+g_{4}+z_{j}} ; q\right)_{\lambda_{j}}}
\end{gathered}
$$

(using $\left.(a ; q)_{\infty} /\left(q^{m} a ; q\right)_{\infty}=(a ; q)_{m}\right)$ and for $q=1$ that

$$
\begin{aligned}
& \frac{\mathscr{C}_{+, 1}(z) \mathscr{C}_{-, 1}(z)}{\mathscr{C}_{+, 1}(z+\lambda) \mathscr{C}_{-, 1}(z+\lambda)} \\
\times \prod_{1 \leq j<k \leq n}\left(1+\frac{\lambda_{j}+\lambda_{k}}{z_{j}+z_{k}}\right)\left(1+\frac{\lambda_{j}-\lambda_{k}}{z_{j}-z_{k}}\right) & \left(-g+z_{j}+z_{k}\right)_{\lambda_{j}+\lambda_{k}}\left(-g+z_{j}-z_{k}\right)_{\lambda_{j}-\lambda_{k}} \\
& \times \frac{\left(1+g+z_{j}+z_{k}\right)_{\lambda_{j}+\lambda_{k}}\left(1+g+z_{j}-z_{k}\right)_{\lambda_{j}-\lambda_{k}}}{\left(1+g_{1}\right.}\left(1+\frac{\lambda_{j}}{z_{j}}\right) \frac{\left(-g_{1}+z_{j},-g_{2}+z_{j},-g_{3}+z_{j},-g_{4}+z_{j}\right)_{\lambda_{j}}}{\left(1+g_{1}+z_{j}, 1+g_{2}+z_{j}, 1+g_{3}+z_{j}, 1+g_{4}+z_{j}\right)_{\lambda_{j}}}
\end{aligned}
$$


(using $\left.\Gamma(a+m) / \Gamma(a)=(a)_{m}\right)$. From (2.23a), (2.23b) and (2.13) it is immediate that the summation formula $(2.22 \mathrm{a}) /(2.22 \mathrm{~b})$ specializes for $n=1$ to the Bailey and Dougall sums in (1.2a) and (1.2b).

Remark. Multidimensional analogues of the Bailey ${ }_{6} \psi_{6}$ and Dougall ${ }_{5} F_{4}$ summation formulas (1.2a), (1.2b) different from the ones in $(2.22 \mathrm{a}) /(2.22 \mathrm{~b})$ were introduced by Gustafson in [Gu1, Gu2] (cf. also Section 4), and recently still another multidimensional version (in two distinct variations) of the ${ }_{6} \psi_{6}$ sum was presented by Schlosser [Sch]. For $g=-1 / 2$ (and $0<q<1$ ), the sum in $(2.22 a) /(2.22 b)$ may be seen as a special case of Schlosser's multiple Bailey sums.

\section{§3. Truncation: Multiple Analogues of Very-Well-Poised Unilateral (Basic) Hypergeometric Series}

In this section the vector $z$ will be specialized in such a way that the multiple Baily/Dougall sum of Section 2.4 truncates to a sum over the dominant cone

$$
\Lambda=\left\{\lambda \in \mathbb{Z}^{n} \mid \lambda_{1} \geq \lambda_{2} \geq \cdots \geq \lambda_{n} \geq 0\right\}
$$

or - for special choice of the parameters - to a finite sum over the sub-alcove

$$
\Lambda_{N}=\left\{\lambda \in \mathbb{Z}^{n} \mid N \geq \lambda_{1} \geq \lambda_{2} \geq \cdots \geq \lambda_{n} \geq 0\right\} \quad \text { (with } N \in N \text { ). }
$$

The resulting summation formulas constitute multiple analogues of the (terminating) very-well-poised Rogers ${ }_{6} \phi_{5}$ and Dougall ${ }_{5} F_{4}$ sums [Ro, Do, GR]. For $0<q<1$ the terms of the multiple series in question are of the form

$$
\begin{aligned}
\Delta_{q}(\lambda)= & q^{\Sigma_{j=1}^{n}\left(1-2 \hat{\rho}_{j}\right) \lambda_{j}} \\
\times & \quad \prod_{1 \leq j<k \leq n}\left(\frac{1-q^{\rho_{j}+\rho_{k}+\lambda_{j}+\lambda_{k}}}{1-q^{\rho_{j}+\rho_{k}}} \frac{1-q^{\rho_{j}-\rho_{k}+\lambda_{j}-\lambda_{k}}}{1-q^{\rho_{j}-\rho_{k}}}\right) \\
& \times \frac{\left(q^{g+\rho_{j}+\rho_{k}} ; q\right)_{\lambda_{j}+\lambda_{k}}\left(q^{g+\rho_{j}-\rho_{k}} ; q\right)_{\lambda_{j}-\lambda_{k}}}{\left(q^{1-g+\rho_{j}+\rho_{k}} ; q\right)_{\lambda_{j}+\lambda_{k}}\left(q^{1-g+\rho_{j}-\rho_{k}} ; q\right)_{\lambda_{j}-\lambda_{k}}} \\
\times & \prod_{1 \leq j \leq n}\left(\frac{1-q^{2 \rho_{j}+2 \lambda_{j}}}{1-q^{2 \rho_{j}}}\right) \frac{\Pi_{r=1}^{4}\left(q^{g_{r}+\rho_{j}} ; q\right)_{\lambda_{j}}}{\Pi_{r=1}^{4}\left(q^{1-g_{r}+\rho_{j}} ; q\right)_{\lambda_{j}}}
\end{aligned}
$$

(corresponding to a Rogers type series) and for $q=1$ we have 


$$
\begin{aligned}
\Delta_{1}(\lambda)=\prod_{1 \leq j<k \leq n}\left(1+\frac{\lambda_{j}+\lambda_{k}}{\rho_{j}+\rho_{k}}\right)\left(1+\frac{\lambda_{j}-\lambda_{k}}{\rho_{j}-\rho_{k}}\right) \\
\quad \times \frac{\left(g+\rho_{j}+\rho_{k}\right)_{\lambda_{j}+\lambda_{k}}\left(g+\rho_{j}-\rho_{k}\right)_{\lambda_{j}-\lambda_{k}}}{\left(1-g+\rho_{j}+\rho_{k}\right)_{\lambda_{j}+\lambda_{k}}\left(1-g+\rho_{j}-\rho_{k}\right)_{\lambda_{j}-\lambda_{k}}} \\
\quad \times \prod_{1 \leq j \leq n}\left(1+\frac{\lambda_{j}}{\rho_{j}}\right) \frac{\Pi_{r=1}^{4}\left(g_{r}+\rho_{j}\right)_{\lambda_{j}}}{\Pi_{r=1}^{4}\left(1-g_{r}+\rho_{j}\right)_{\lambda_{j}}}
\end{aligned}
$$

(corresponding to a Dougall type series), where $\rho_{j}$ and $\hat{\rho}_{j}$ are given by (2.4).

\subsection{Multiple Rogers ${ }_{6} \phi_{5}$ and Dougall ${ }_{5} F_{4}$ Sums}

It is not so difficult to infer that the terms of the Bailey/Dougall sum (2.22a), (2.22b) become zero for $\lambda \in Z^{n} \backslash \Lambda$ (with $\Lambda$ given by (3.1)) if one sets $z=-\rho$. Indeed, when $z_{j}=-\rho_{j}(2.4)(j=1, \cdots, n)$ the factors $\left(q^{1+g+z_{j}-z_{j+1}}\right.$; $q)_{\lambda_{j}-\lambda_{j+1}},\left(1+g+z_{j}-z_{j+1}\right)_{\lambda_{j}-\lambda_{j+1}}$ and $\left(q^{1+g_{a}+z_{n}} ; q\right)_{\lambda_{n}},\left(1+g_{a}+z_{n}\right)_{\lambda_{n}}$ in the denominators of $(2.23 \mathrm{a}),(2.23 \mathrm{~b})$ give rise to a zero for $\lambda_{j}<\lambda_{j+1}$ and $\lambda_{n}<0$, respectively. Simplification of the r.h.s. of $(2.22 \mathrm{a}) /(2.22 \mathrm{~b})$ (for $z=-\rho$ the factors $\mathscr{C}_{+. q}(\rho)$ and $1 / \hat{\mathscr{C}}_{+. q}(\hat{\rho})$ cancel each other) and reflection of the parameters in the origin $\left(g \rightarrow-g, g_{r} \rightarrow-g_{r}\right)$ so as to avoid the excessive appearance of minus signs, finally leads us to the following summation theorem.

Theorem 2. Let $0<q \leq 1$. For parameters subject to the condition

$$
\operatorname{Re}\left(1-2(n-j) g-g_{1}-g_{2}-g_{3}-g_{4}\right)>0
$$

$(j=1, \cdots, n)$, one has that

$$
\sum_{\lambda \in \Lambda} \Delta_{q}(\lambda)=\mathscr{N}_{q, \Lambda}
$$

with

$$
\begin{aligned}
\mathscr{N}_{q . \Lambda}= & \prod_{1 \leq j<k \leq n} \frac{\left(q^{1+\rho_{j}+\rho_{k}}, q^{1+\rho_{j}-\rho_{k}}, q^{1+g-\hat{\rho}_{j}-\hat{\rho}_{k}}, q^{1+g-\hat{\rho}_{j}+\hat{\rho}_{k}} ; q\right)_{\infty}}{\left(q^{1-g+\rho_{j}+\rho_{k}}, q^{1-g+\rho_{j}-\rho_{k}}, q^{1-\hat{\rho}_{j}-\hat{\rho}_{k}}, q^{1-\hat{\rho}_{j}+\hat{\rho}_{k}} ; q\right)_{\infty}} \\
& \times \prod_{1 \leq j \leq n} \frac{\left(q^{1+2 \rho_{j}} ; q\right)_{\infty} \Pi_{r=1}^{4}\left(q^{1+\hat{g}_{r}-\hat{\rho}_{j}} ; q\right)_{\infty}}{\left(q^{1-2 \hat{\rho}_{j}} ; q\right)_{\infty} \Pi_{r=1}^{4}\left(q^{1-g_{r}+\rho_{j}} ; q\right)_{\infty}}
\end{aligned}
$$

for $0<q<1$ and 


$$
\begin{aligned}
\mathscr{N}_{1, \Lambda}= & \prod_{1 \leq j<k \leq n} \frac{\Gamma\left(1-g+\rho_{j}+\rho_{k}\right) \Gamma\left(1-g+\rho_{j}-\rho_{k}\right)}{\Gamma\left(1+\rho_{j}+\rho_{k}\right) \Gamma\left(1+\rho_{j}-\rho_{k}\right)} \\
& \times \frac{\Gamma\left(1-\hat{\rho}_{j}-\hat{\rho}_{k}\right) \Gamma\left(1-\hat{\rho}_{j}+\hat{\rho}_{k}\right)}{\Gamma\left(1+g-\hat{\rho}_{j}-\hat{\rho}_{k}\right) \Gamma\left(1+g-\hat{\rho}_{j}+\hat{\rho}_{k}\right)} \\
& \times \prod_{1 \leq j \leq n} \frac{\Gamma\left(1-2 \hat{\rho}_{j}\right) \Pi_{r=1}^{4} \Gamma\left(1-g_{r}+\rho_{j}\right)}{\Gamma\left(1+2 \rho_{j}\right) \Pi_{r=1}^{4} \Gamma\left(1+\hat{g}_{r}-\hat{\rho}_{j}\right)} .
\end{aligned}
$$

Moreover, the series on the l.h.s. of (3.5) converges in absolute value.

By canceling common terms in numerator and denominator the evaluation constants (3.6a) and (3.6b) may be rewritten as

$$
\begin{array}{r}
\mathscr{N}_{q, \Lambda}=\prod_{1 \leq j \leq n} \frac{\left(q^{1+(2 n-j-1) g+2 g_{a}} ; q\right)_{\infty}}{\left(q^{1-(2 n-j-1) g-g_{a}-g_{b}-g_{c}-g_{d}} ; q\right)_{\infty}} \\
\times \frac{\prod_{\substack{1 \leq r<s \leq 4 \\
r, s \neq a}}\left(q^{1-(n-j) g-g_{r}-g_{s}} ; q\right)_{\infty}}{\prod_{\substack{1 \leq r \leq 4 \\
r \neq a}}\left(q^{1+(n-j) g+g_{a}-g_{r}} ; q\right)_{\infty}}
\end{array}
$$

$(0<q<1)$ and

$$
\begin{array}{r}
\mathscr{N}_{1 . \Lambda}=\prod_{1 \leq j \leq n} \frac{\Gamma\left(1-(2 n-j-1) g-g_{a}-g_{b}-g_{c}-g_{d}\right)}{\Gamma\left(1+(2 n-j-1) g+2 g_{a}\right)} \\
\times \frac{\Pi_{\substack{1 \leq r \leq 4 \\
r \neq a}} \Gamma\left(1+(n-j) g+g_{a}-g_{r}\right)}{\prod_{\substack{1 \leq r<s \leq 4 \\
r, s \neq a}} \Gamma\left(1-(n-j) g-g_{r}-g_{s}\right)},
\end{array}
$$

respectively. When $n=1$ the summation formula in Theorem 2 reduces for $0<q<1$ to the nonterminating Rogers sum (cf. [Ro, GR])

$$
\begin{aligned}
& \sum_{\lambda \in N} q^{\left(1-g_{a}-g_{b}-g_{c}-g_{d}\right) \lambda}\left(\frac{1-q^{2 g_{a}+2 \lambda}}{1-q^{2 g_{a}}}\right) \prod_{1 \leq r \leq 4} \frac{\left(q^{g_{r}+g_{a}} ; q\right)_{\lambda}}{\left(q^{1-g_{r}+g_{a}} ; q\right)_{\lambda}}
\end{aligned}
$$

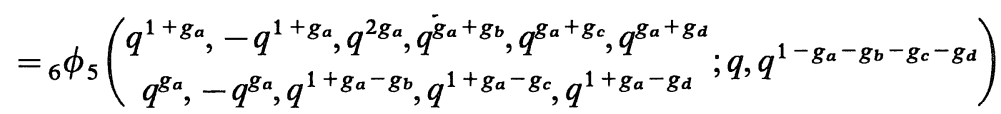

$$
\begin{aligned}
& =\frac{\left(q^{1+2 g_{a}}, q^{1-g_{b}-g_{c}}, q^{1-g_{b}-g_{d}}, q^{1-g_{c}-g_{d}} ; q\right)_{\infty}}{\left(q^{1+g_{a}-g_{b}}, q^{1+g_{a}-g_{c}}, q^{1+g_{a}-g_{d}}, q^{1-g_{a}-g_{b}-g_{c}-g_{d}} ; q\right)_{\infty}}
\end{aligned}
$$


and for $q=1$ to the nonterminating Dougall sum (cf. [Do, GR])

$$
\begin{aligned}
& \sum_{\lambda \in N}\left(1+\frac{\lambda}{g_{a}}\right)_{1} \prod_{1 \leq r \leq 4} \frac{\left(g_{r}+g_{a}\right)_{\lambda}}{\left(1-g_{r}+g_{a}\right)_{\lambda}} \\
= & { }_{5} F_{4}\left(\begin{array}{c}
1+g_{a}, 2 g_{a}, g_{a}+g_{b}, g_{a}+g_{c}, g_{a}+g_{d} \\
g_{a}, 1+g_{a}-g_{b}, 1+g_{a}-g_{c}, 1+g_{a}-g_{d}
\end{array}\right) \\
= & \frac{\Gamma\left(1+g_{a}-g_{b}\right) \Gamma\left(1+g_{a}-g_{c}\right) \Gamma\left(1+g_{a}-g_{d}\right) \Gamma\left(1-g_{a}-g_{b}-g_{c}-g_{d}\right)}{\Gamma\left(1+2 g_{a}\right) \Gamma\left(1-g_{b}-g_{c}\right) \Gamma\left(1-g_{b}-g_{d}\right) \Gamma\left(1-g_{c}-g_{d}\right)},
\end{aligned}
$$

where the parameters are assumed to satisfy the convergence condition $\operatorname{Re}\left(1-g_{a}-g_{b}-g_{c}-g_{d}\right)>0$.

\subsection{Terminating Multiple Rogers ${ }_{6} \phi_{5}$ and Dougall ${ }_{5} F_{4}$ Sums}

When the parameters in the Rogers/Dougall type sum of Theorem 2 are chosen in such a way that $(n-1) g+g_{a}+g_{b}+N=0$ with $N \in N$, then the series on the 1.h.s. terminates as the terms $\Delta_{q}(\lambda)$ (3.3a), (3.3b) become zero for $\lambda \in \Lambda \backslash \Lambda_{N}$ (where $\Lambda_{N}$ is given by (3.2)). This is because we now pick up a zero from the factor $\left(q^{g_{b}+\rho_{1}} ; q\right)_{\lambda_{1}}$ or $\left(g_{b}+\rho_{1}\right)_{\lambda_{1}}$ in the numerator when $\lambda_{1}>N$. As a consequence, the 1.h.s. of (3.5) becomes a rational expression in $q, q^{g}, q^{g_{r}}$ (for $q \neq 1$ ) or in $g, g_{r}$ (for $q=1$ ) and the same must be true for the corresponding r.h.s. Indeed, for the parameters subject to the above truncation condition the infinite products entering $\mathscr{N}_{\Lambda}(3.6 \mathrm{a}),(3.6 \mathrm{~b})$ may be reduced to finite products by canceling common factors in the numerator and denominator. This way one arrives at an expression for the r.h.s. that can be written as

$$
\begin{aligned}
\mathscr{N}_{q, \Lambda_{N}} & =\prod_{1 \leq j<k \leq n} \frac{\left(q^{1+\rho_{J}+\rho_{k}} ; q\right)_{N}}{\left(q^{1-g+\rho_{J}+\rho_{k}} ; q\right)_{N}} \prod_{1 \leq j \leq n} \frac{\left(q^{1+2 \rho_{J}}, q^{1+\hat{g}_{b}-\hat{\rho}_{j}} ; q\right)_{N}}{\left(q^{1-g_{c}+\rho_{j}}, q^{1-g_{d}+\rho_{j}} ; q\right)_{N}} \\
& =\prod_{1 \leq j<k \leq n} \frac{\left(q^{1+g-\hat{\rho}_{j}-\hat{\rho}_{k}} ; q\right)_{-N}}{\left(q^{1-\hat{\rho}_{j}-\hat{\rho}_{k}} ; q\right)_{-N}} \prod_{1 \leq j \leq n} \frac{\left(q^{1+\hat{g}_{c}-\hat{\rho}_{j}}, q^{1+\hat{g}_{d}-\hat{\rho}_{j}} ; q\right)_{-N}}{\left(q^{1-2 \hat{\rho}_{j}}, q^{1-g_{b}+\rho_{j}} ; q\right)_{-N}}
\end{aligned}
$$

for $q \neq 1$ and as

$$
\begin{aligned}
\mathscr{N}_{1, \Lambda_{N}} & =\prod_{1 \leq j<k \leq n} \frac{\left(1+\rho_{j}+\rho_{k}\right)_{N}}{\left(1-g+\rho_{j}+\rho_{k}\right)_{N}} \prod_{1 \leq j \leq n} \frac{\left(1+2 \rho_{j}, 1+\hat{g}_{b}-\hat{\rho}_{j}\right)_{N}}{\left(1-g_{c}+\rho_{j}, 1-g_{d}+\rho_{j}\right)_{N}} \\
& =\prod_{1 \leq j<k \leq n} \frac{\left(1+g-\hat{\rho}_{j}-\hat{\rho}_{k}\right)_{-N}}{\left(1-\hat{\rho}_{j}-\hat{\rho}_{k}\right)_{-N}} \prod_{1 \leq j \leq n} \frac{\left(1+\hat{g}_{c}-\hat{\rho}_{j}, 1+\hat{g}_{d}-\hat{\rho}_{j}\right)_{-N}}{\left(1-2 \hat{\rho}_{j}, 1-g_{b}+\rho_{j}\right)_{-N}}
\end{aligned}
$$


for $q=1$. For the equality of the expressions on the first line and the second line of the formulas (3.9a), (3.9b) it is essential that the parameters satisfy the truncation condition $(n-1) g+g_{a}+g_{b}+N=0$. The corresponding expression for the r.h.s. of the terminating multiple Rogers/Dougall summation formula becomes somewhat more symmetric if we combine the both representations for $\mathscr{N}_{q, \Lambda_{N}}$ into a single expression for $\mathcal{N}_{q, \Lambda_{N}}^{2}$.

Theorem 3. Let $N \in N$. For parameters subject to the truncation condition

$$
(n-1) g+g_{a}+g_{b}+N=0
$$

(which is to be read modulo $2 \pi / i \log q$ for $q \neq 1$ ), one has that

$$
\sum_{\lambda \in \Lambda_{N}} \Delta_{q}(\lambda)=\mathscr{N}_{q, \Lambda_{N}}
$$

with

$$
\begin{aligned}
\mathscr{N}_{q, \Lambda_{N}}^{2}= & \prod_{1 \leq j<k \leq n} \frac{\left(q^{1+\rho_{j}+\rho_{k}} ; q\right)_{N}}{\left(q^{1-g+\rho_{j}+\rho_{k}} ; q\right)_{N}} \frac{\left(q^{1+g-\hat{\rho}_{j}-\hat{\rho}_{k}} ; q\right)_{-N}}{\left(q^{1-\hat{\rho}_{j}-\hat{\rho}_{k}} ; q\right)_{-N}} \\
& \times \prod_{1 \leq j \leq n} \frac{\left(q^{1+2 \rho_{j}}, q^{1+\hat{g}_{b}-\hat{\rho}_{j}} ; q\right)_{N}}{\left(q^{1-2 \hat{\rho}_{j}}, q^{1-g_{b}+\rho_{j}} ; q\right)_{-N}} \frac{\left(q^{1+\hat{g}_{c}-\hat{\rho}_{j}}, q^{1+\hat{g}_{d}-\hat{\rho}_{j}} ; q\right)_{-N}}{\left(q^{1-g_{c}+\rho_{j}} ; q^{1-g_{d}+\rho_{j}} ; q\right)_{N}}
\end{aligned}
$$

for $q \neq 1$ and

$$
\begin{aligned}
\mathscr{N}_{1, \Lambda_{N}}^{2}= & \prod_{1 \leq j<k \leq n} \frac{\left(1+\rho_{j}+\rho_{k}\right)_{N}}{\left(1-g+\rho_{j}+\rho_{k}\right)_{N}} \frac{\left(1+g-\hat{\rho}_{j}-\hat{\rho}_{k}\right)_{-N}}{\left(1-\hat{\rho}_{j}-\hat{\rho}_{k}\right)_{-N}} \\
& \times \prod_{1 \leq j \leq n} \frac{\left(1+2 \rho_{j}, 1+\hat{g}_{b}-\hat{\rho}_{j}\right)_{N}}{\left(1-2 \hat{\rho}_{j}, 1-g_{b}+\rho_{j}\right)_{-N}} \frac{\left(1+\hat{g}_{c}-\hat{\rho}_{j}, 1+\hat{g}_{d}-\hat{\rho}_{j}\right)_{-N}}{\left(1-g_{c}+\rho_{j}, 1-g_{d}+\rho_{j}\right)_{N}} .
\end{aligned}
$$

The summation formula (3.11) holds as a rational identity in $q^{g}, q^{g_{a}}, q^{g_{b}}, q^{g_{c}}, q^{g_{d}}$ and $q($ for $q \neq 1)$ or in $g, g_{a}, g_{b}, g_{c}, g_{d}$ (for $\left.q=1\right)$, subject to the relation (3.10).

By further cancellation of common factors in the numerator and denominator the evaluation constants (3.9a), (3.9b) may be rewritten as

$$
\begin{aligned}
\mathscr{N}_{q, \Lambda_{N}} & =\prod_{1 \leq j \leq n} \frac{\left(q^{1+(2 n-j-1) g+2 g_{a}}, q^{1-(n-j) g-g_{c}-g_{d}} ; q\right)_{N}}{\left(q^{1+(n-j) g+g_{a}-g_{c}}, q^{1+(n-j) g+g_{a}-g_{d}} ; q\right)_{N}} \\
& =\prod_{1 \leq j \leq n} \frac{\left(q^{1-(n-j) g-\hat{g}_{a}+\hat{g}_{c}}, q^{1-(n-j) g-\hat{g}_{a}+\hat{g}_{d}} ; q\right)_{-N}}{\left(q^{1-(2 n-j-1) g-2 \hat{g}_{a}}, q^{1+(n-j) g+\hat{g}_{c}+\hat{g}_{d}} ; q\right)_{-N}}
\end{aligned}
$$


$(q \neq 1)$ and

$$
\begin{aligned}
\mathscr{N}_{1, \Lambda_{N}} & =\prod_{1 \leq j \leq n} \frac{\left(1+(2 n-j-1) g+2 g_{a}, 1-(n-j) g-g_{c}-g_{d}\right)_{N}}{\left(1+(n-j) g+g_{a}-g_{c}, 1+(n-j) g+g_{a}-g_{d}\right)_{N}} \\
& =\prod_{1 \leq j \leq n} \frac{\left(1-(n-j) g-\hat{g}_{a}+\hat{g}_{c}, 1-(n-j) g-\hat{g}_{a}+\hat{g}_{d}\right)_{-N}}{\left(1-(2 n-j-1) g-2 \hat{g}_{a}, 1+(n-j) g+\hat{g}_{c}+\hat{g}_{d}\right)_{-N}}
\end{aligned}
$$

respectively. When $n=1$ the summation formula of Theorem 3 reduces to the terminating Rogers sum (cf. [Ro, GR])

$$
\begin{aligned}
& \sum_{\lambda \in\{0, \cdots, N\}} q^{\left(1-g_{a}-g_{b}-g_{c}-g_{d}\right) \lambda}\left(\frac{1-q^{2 g_{a}+2 \lambda}}{1-q^{2 g_{a}}}\right) \prod_{1 \leq r \leq 4} \frac{\left(g^{g_{r}+g_{a}} ; q\right)_{\lambda}}{\left(q^{1-g_{r}+g_{a}} ; q\right)_{\lambda}}
\end{aligned}
$$

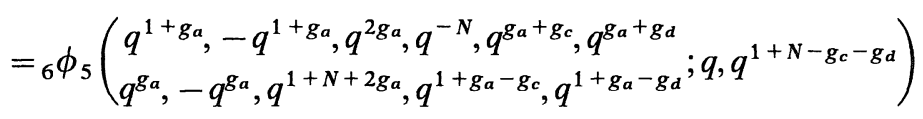

$$
\begin{aligned}
& =\frac{\left(q^{1+2 g_{a}}, q^{1-g_{c}-g_{d}} ; q\right)_{N}}{\left(q^{1+g_{a}-g_{c}}, q^{1+g_{a}-g_{d}} ; q\right)_{N}}=\frac{\left(q^{1-\hat{g}_{a}+\hat{g}_{c}}, q^{1-\hat{g}_{a}+\hat{g}_{d}} ; q\right)_{-N}}{\left(q^{1-2 \hat{g_{a}}}, q^{1+\hat{g}_{c}+\hat{g}_{d}} ; q\right)_{-N}}
\end{aligned}
$$

for $q \neq 1$ and to the terminating Dougall sum (cf. [Do, GR])

$$
\begin{aligned}
& \sum_{\lambda \in\{0, \cdots, N\}}\left(1+\frac{\lambda}{g_{a}}\right) \prod_{1 \leq r \leq 4} \frac{\left(g_{r}+g_{a}\right)_{\lambda}}{\left(1-g_{r}+g_{a}\right)_{\lambda}} \\
= & { }_{5} F_{4}\left(\begin{array}{c}
1+g_{a}, 2 g_{a},-N, g_{a}+g_{c}, g_{a}+g_{d} ; 1 \\
g_{a}, 1+N+2 g_{a}, 1+g_{a}-g_{c}, 1+g_{a}-g_{d}
\end{array}\right) \\
= & \frac{\left(1+2 g_{a}, 1-g_{c}-g_{d}\right)_{N}}{\left(1+g_{a}-g_{c}, 1+g_{a}-g_{d}\right)_{N}}=\frac{\left(1-\hat{g}_{a}+\hat{g}_{c}, 1-\hat{g}_{a}+\hat{g}_{d}\right)_{-N}}{\left(1-2 \hat{g}_{a}, 1+\hat{g}_{c}+\hat{g}_{d}\right)_{-N}}
\end{aligned}
$$

for $q=1$, where the parameters are assumed to satisfy the truncation condition $g_{a}+g_{b}+N=0$ (modulo $2 \pi / i \log q$ ).

Let us conclude with an important application of Theorem 3 to the theory of (basic) hypergeometric orthogonal polynomials in several variables. In [DS] a multivariable generalization of the $(q$-) Racah polynomials $[\mathrm{AW}, \mathrm{W}]$ was studied. For $q \neq 1$ the multivariable polynomials of interest amount to the $B C$ type Askey-Wilson polynomials due (for special parameters) to Macdonald [Ma1] and (for general parameters) to Koornwinder [Ko], whereas the degenerate case $q=1$ corresponds to the multivariable Wilson polynomials considered in [D]. In [Ma1, Ko, D] the parameter domains were chosen 
such that the relevant orthogonality measures are continuous. The paper [DS] focuses on a different parameter regime for which the orthogonality measure becomes purely discrete and finitely supported on the grid points $\rho_{a}+\lambda, \lambda \in \Lambda_{N}$, with weights that are given by $\Delta_{q}(\lambda)$ (3.3a), (3.3b). Among other things, [DS] presents product formulas for the (squared) norms of the multivariable $(q$-)Racah polynomials (with respect to the corresponding discrete inner product) in terms of the (squared) norm of the unit polynomial. The sum in Theorem 3 provides a product formula for the latter norm (i.e. the squared norm of the unit polynomial) and thus completes the solution of the orthonormalization problem for the multivariable $(q$-) Racah polynomials in product form. For $n=1$ the resulting product formulas reduce to the norm formulas for the one-variable $(q$-)Racah polynomials presented by Askey and Wilson [AW, W].

Remark. Multidimensional analogues of the Dougall ${ }_{5} F_{4}$ sum (3.8b), (3.14b) and the Rogers ${ }_{6} \phi_{5}$ sum (3.8a), (3.14a) different from those considered in Theorem 2 and Theorem 3 can be found in [Ho] $\left({ }_{5} F_{4}\right.$ type) and [M1, M2, LM, Sch] $\left({ }_{6} \phi_{5}\right.$ type).

\section{§4. Proof of Theorem $\mathbb{1}$}

We will prove Theorem 1 by deducing a recurrence relation for the Macdonald type sum (2.11) using a technique due to Gustafson, who applied it to evaluate a Selberg type multivariable generalization of the Askey-Wilson integral [Gu3]. Essential ingredient in the derivation of the recurrence relation is a multiple summation formula taken from [Gu2, Gu4]. Let the function $\Delta_{q}^{G}(\mathbf{x})$ be given for $0<q<1$ by

$$
\begin{aligned}
\Delta_{q}^{G}(\mathbf{x})= & \prod_{1 \leq j<k \leq n} \frac{1}{\left(q^{1+x_{j}+x_{k}}, q^{1+x_{j}-x_{k}}, q^{1-x_{j}+x_{k}}, q^{1-x_{j}-x_{k}} ; q\right)_{\infty}} \\
& \times \prod_{1 \leq j \leq n} \frac{\prod_{r=1}^{2 n+2}\left(q^{1+g_{r}+x_{j}} ; q^{1+g_{r}-x_{j}} ; q\right)_{\infty}}{\left(q^{1+2 x_{j}} ; q^{1-2 x_{j}} ; q\right)_{\infty}}
\end{aligned}
$$

and for $q=1$ by

$$
\Delta_{1}^{G}(\mathbf{x})=\prod_{1 \leq j<k \leq n} \Gamma\left(1+x_{j}+x_{k}\right) \Gamma\left(1+x_{j}-x_{k}\right) \Gamma\left(1-x_{j}+x_{k}\right) \Gamma\left(1-x_{j}-x_{k}\right)
$$




$$
\times \prod_{1 \leq j \leq n} \frac{\Gamma\left(1+2 x_{j}\right) \Gamma\left(1-2 x_{j}\right)}{\prod_{r=1}^{2 n+2} \Gamma\left(1+g_{r}+x_{j}\right) \Gamma\left(1+g_{r}-x_{j}\right)} .
$$

Then one has for parameters satisfying the condition $\operatorname{Re}\left(1+g_{1}+\cdots+g_{2 n+2}\right)>0$ that $[\mathrm{Gu} 2, \mathrm{Gu} 4]$

$$
\begin{aligned}
& \sum_{\lambda \in Z^{n}} \Delta_{q}^{G}(z+\lambda) \\
& = \begin{cases}\frac{(q ; q)_{\infty}^{n} \Pi_{1 \leq r<s \leq 2 n+2}\left(q^{1+g_{r}+g_{s}} ; q\right)_{\infty}}{\left(q^{1+g_{1}+\cdots+g_{2 n+2}} ; q\right)_{\infty}} & \text { for } 0<q<1 \\
\frac{\Gamma\left(1+g_{1}+\cdots+g_{2 n+2}\right)}{\Pi_{1 \leq r<s \leq 2 n+2} \Gamma\left(1+g_{r}+g_{s}\right)} & \text { for } q=1\end{cases}
\end{aligned}
$$

(where $z \in C^{n}$ is assumed to satisfy the genericity condition that the combinations $z_{j}+z_{k} \quad z_{j}-z_{k} \quad(1 \leq j<k \leq n)$ and $2 z_{j} \quad(1 \leq j \leq n)$ are nonzero modulo $\Omega_{q}$ (2.9)). Moreover, the series on the 1.h.s. of (4.3) converges in absolute value (this may be verified in a similar manner as was done in the appendix at the end of the paper for the Macdonald type series on the 1.h.s. of (2.11)). For $n=1$, Gustafson's sum in (4.3) reduces to the summation formula (1.1a), (1.1b). In other words, the Gustafson sum (4.3) amounts to a multidimensional generalization of the (in essence) Bailey/Dougall sum $(1.1 \mathrm{a}) /(1.1 \mathrm{~b})$ that is different from the Macdonald type generalization described by Theorem 1 (cf. the remark below).

Let us abbreviate the Macdonald type sum $\Sigma_{\lambda \in Z^{n}} 1 /\left(\mathscr{C}_{+, q}(z+\lambda) \mathscr{C}_{+, q}(-z\right.$ $-\lambda)$ ) by $S_{n}\left(g, g_{r} ; q\right)$ and consider the double sum (for $0<q<1$ )

$$
\begin{aligned}
& \sum_{\lambda \in \mathbf{Z}^{n}} \sum_{\mu \in \mathbf{Z}^{n-1}} \prod_{1 \leq j<k \leq n}\left(\frac{1}{\left(q^{1+z_{j}+z_{k}+\lambda_{J}+\lambda_{k}}, q^{1+z_{J}-z_{k}+\lambda_{J}-\lambda_{k}} ; q\right)_{\infty}}\right. \\
&\left.\times \frac{1}{\left(q^{1-z_{J}+z_{k}-\lambda_{J}+\lambda_{k}}, q^{1-z_{J}-z_{k}-\lambda_{j}-\lambda_{k}} ; q\right)_{\infty}}\right) \\
& \quad \times \prod_{j=1}^{n} \frac{\prod_{r=1}^{4}\left(q^{1+g_{r}+z_{J}+\lambda_{j}}, q^{1+g_{r}-z_{j}-\lambda_{j}} ; q\right)_{\infty}}{\left(q^{1+2 z_{J}+2 \lambda_{J}}, q^{1-2 z_{J}-2 \lambda_{j}} ; q\right)_{\infty}} \\
& \quad \times \prod_{1 \leq j<k \leq n-1}\left(\frac{1}{\left(q^{1+z_{J}+z_{k}+\mu_{j}+\mu_{k}}, q^{1+z_{J}-z_{k}+\mu_{J}-\mu_{k}} ; q\right)_{\infty}}\right.
\end{aligned}
$$




$$
\begin{gathered}
\left.\times \frac{1}{\left(q^{1-z_{j}+z_{k}-\mu_{j}+\mu_{k}}, q^{1-z_{j}-z_{k}-\mu_{j}-\mu_{k}} ; q\right)_{\infty}}\right) \\
\times \prod_{k=1}^{n-1} \frac{1}{\left(q^{1+2 z_{k}+2 \mu_{k}}, q^{1-2 z_{k}-2 \mu_{k}} ; q\right)_{\infty}} \\
\times \prod_{j=1}^{n} \prod_{k=1}^{n-1}\left(\left(q^{1+g / 2+z_{j}+z_{k}+\lambda_{j}+\mu_{k}}, q^{1+g / 2+z_{j}-z_{k}+\lambda_{j}-\mu_{k}} ; q\right)_{\infty}\right. \\
\left.\times\left(q^{1+g / 2-z_{j}+z_{k}-\lambda_{j}+\mu_{k}}, q^{1+g / 2-z_{j}-z_{k}-\lambda_{j}-\mu_{k}} ; q\right)_{\infty}\right) .
\end{gathered}
$$

We can express this double sum in terms of $S_{n}\left(g, g_{r} ; q\right)$ by evaluating the inner sum (in $\mu$ ) with the aid of Gustafson's formula (4.3). Alternatively, we may also reverse the order of summation in $\lambda$ and $\mu$ (using the convergence in absolute value) and instead apply Gustafson's formula (4.3) to the (now inner) sum over $\lambda$; this yields an expression for the double sum in terms of $S_{n-1}\left(g, g_{r}+g / 2 ; q\right)$. Comparing the two expressions for the double sum thus obtained entails the recurrence relation

$$
S_{n}\left(g, g_{r} ; q\right)=\frac{\left(q, q^{1+n g} ; q\right)_{\infty} \Pi_{1 \leq r<s \leq 4}\left(q^{1+g_{r}+g_{s}} ; q\right)_{\infty}}{\left(q^{1+g}, q^{1+(n-1) g+g_{1}+g_{2}+g_{3}+g_{4}} ; q\right)_{\infty}} S_{n-1}\left(g, g_{r}+g / 2 ; q\right),
$$

which by induction on $n$ starting from the known value for $n=1$ (taken from the r.h.s. of (1.1a)) produces

$$
S_{n}\left(g, g_{r} ; q\right)=\prod_{1 \leq j \leq n} \frac{\left(q, q^{1+j g} ; q\right)_{\infty} \Pi_{1 \leq r<s \leq 4}\left(q^{1+(n-j) g+g_{r}+g_{s}} ; q\right)_{\infty}}{\left(q^{1+g}, q^{1+(2 n-j-1) g+g_{1}+g_{2}+g_{3}+g_{4}} ; q\right)_{\infty}} .
$$

For $q=1$ the derivation is quite similar and leads us starting from the $q=1$ degeneration of the double sum (4.4) via Gustafson's sum (4.3) to the recurrence relation

$$
\begin{aligned}
& S_{n}\left(g, g_{r} ; 1\right) \\
= & \frac{\Gamma(1+g) \Gamma\left(1+(n-1) g+g_{1}+g_{2}+g_{3}+g_{4}\right)}{\Gamma(1+n g) \Pi_{1 \leq r<s \leq 4} \Gamma\left(1+g_{r}+g_{s}\right)} S_{n-1}\left(g, g_{r}+g / 2 ; 1\right),
\end{aligned}
$$

which - when combined with the know value for $n=1$ (from the r.h.s. of (1.1b)) - entails

$$
S_{n}\left(g, g_{r} ; 1\right)=\prod_{1 \leq j \leq n} \frac{\Gamma(1+g) \Gamma\left(1+(2 n-j-1) g+g_{1}+g_{2}+g_{3}+g_{4}\right)}{\Gamma(1+j g) \Pi_{1 \leq r<s \leq 4} \Gamma\left(1+(n-j) g+g_{r}+g_{s}\right)} .
$$


The theorem now follows from the observation that $S_{n}\left(g, g_{r} ; q\right)(4.6),(4.8)$ may be rewritten in the form given by the r.h.s. of (2.11) (see Eq. (2.13)).

Remark. Division of Gustafson's sum (4.3) from [Gu4, Section 2] by the middle term $\Delta_{q}^{G}(z)$ (corresponding to $\lambda=0$ ), turns it into a multidimensional generalization of the Bailey and Dougall sums (1.2a) and (1.2b) that was introduced in Theorem 5.1 (for $0<q<1$ ) and Theorem 8.3 (for $q=1$ ) of [Gu2]. These multiple Bailey/Dougall sums are different from those described in Section 2.4 and are (for $0<q<1$ ) also different from (but reminiscent of) the multiple Bailey sums considered by Schlosser [Sch].

\section{Appendix: Proof of Absolute Convergence Multiple (Basic) Hypergeometric Series}

In this appendix it is shown that the Macdonald type series (2.11), the generalized Aomoto-Ito series (2.14), the multiple Bailey/Dougall series (2.22a), (2.22b) and the multiple Rogers/Dougall series (3.5) all converge in absolute value. Similar convergence proofs can be found e.g. in [Ka, I, Ma2].

Clearly it suffices to prove the absolute convergence for either one of (2.11), (2.14) or $(2.22 \mathrm{a}) /(2.22 \mathrm{~b})$, as the series in question are equivalent up to multiplication by overall factors and the convergence of the Rogers/Dougall series (3.5) follows immediately from the convergence of the Bailey/Dougall series $(2.22 \mathrm{a}) /(2.22 \mathrm{~b})$ upon specialization of $z$ to the value $-\rho$ (and keeping in mind that we have reflected the parameters $g, g_{r}$ to $-g,-g_{r}$ thus giving rise to the minus signs in the convergence condition (3.4)). In view of the fact that the function $1 /\left(\mathscr{C}_{+, q}(\mathbf{x}) \mathscr{C}_{+, q}(-\mathbf{x})\right)$ is permutation-invariant and even in the components $x_{1}, \cdots, x_{n}$, we conclude from the representation in (2.11) that it is sufficient to show that the restricted sum over the dominant cone $\Lambda$ (3.1) converges absolutely (the cone $\Lambda$ is a fundamental domain for the action of the (Weyl) group that permutes and flips the signs of the components of $\lambda \in Z^{n}$ ). In terms of the Aomoto-Ito type representation of our series given by (2.14) this means that it suffices to demonstrate that

$$
\sum_{\lambda \in \Lambda} \frac{1}{\left|\mathscr{C}_{+, q}(z+\lambda) \mathscr{C}_{-, q}(z+\lambda)\right|}<\infty .
$$

For $0<q<1$, we see from the explicit formula for the terms in (2.16) that the series on the 1.h.s. of (A.1) converges provided 


$$
\sum_{\lambda \in \Lambda}\left|q^{\sum_{j=1}^{n}\left(1+2 \hat{\rho}_{j}\right) \lambda_{j}}\right|<\infty
$$

which is the case when $\operatorname{Re}\left(1+2 \hat{\rho}_{j}\right)=\operatorname{Re}\left(1+2(n-j) g+g_{1}+g_{2}+g_{3}+g_{4}\right)>0$ for $j=1, \cdots, n$. Here we have used that the other factors of the type

$$
\left|1-q^{2 z_{j}+2 \lambda_{j}}\right|, \quad\left|\frac{\left(q^{1+g_{r}+z_{j}+\lambda_{j}} ; q\right)_{\infty}}{\left(q^{-g_{r}+z_{j}+\lambda_{j}} ; q\right)_{\infty}}\right|
$$

and

$$
\left|1-q^{z_{j} \pm z_{k}+\lambda_{j} \pm \lambda_{k}}\right|, \quad\left|\frac{\left(q^{1+g+z_{j} \pm z_{k}+\lambda_{j} \pm \lambda_{k}} ; q\right)_{\infty}}{\left(q^{-g+z_{j} \pm z_{k}+\lambda_{j} \pm \lambda_{k}} ; q\right)_{\infty}}\right|
$$

entering the terms of (A.1) (cf. (2.16)) are bounded on the dominant cone $\Lambda$ (3.1) (one has that $\lim _{x \rightarrow+\infty}\left(a q^{x} ; q\right)_{\infty} /\left(b q^{x}, q\right)_{\infty}=1$ ).

To analyze the convergence of (A.1) for the degenerate case $q=1$ we apply the gamma function asymptotics $\Gamma(a+x) / \Gamma(b+x)=x^{a-b}(1+O(1 / x))$ for $x$ $\rightarrow+\infty$ to the factors of the explicit formula for $1 /\left(\mathscr{C}_{+, 1}(\mathbf{x}) \mathscr{C}_{-, 1}(\mathbf{x})\right)$ in (2.18). This entails that for $q=1$ the series on the 1.h.s. of (A.1) converges when

$$
\begin{aligned}
& \sum_{\lambda \in \Lambda}\left(\prod_{1 \leq j<k \leq n}\left(1+\lambda_{j}+\lambda_{k}\right)^{-2 \operatorname{Re}(g)}\left(1+\lambda_{j}-\lambda_{k}\right)^{-2 \operatorname{Re}(g)}\right. \\
&\left.\times \prod_{1 \leq j \leq n}\left(1+\lambda_{j}\right)^{-3-2 \operatorname{Re}\left(g_{1}+g_{2}+g_{3}+g_{4}\right)}\right)<\infty .
\end{aligned}
$$

(The ratio of $1 /\left|\mathscr{C}_{+, 1}(z+\lambda) \mathscr{C}_{-, 1}(z+\lambda)\right|$ and the corresponding term from (A.3) remains bounded when $\lambda$ runs through the dominant cone $\Lambda$ (3.1).) Similarly, the series on the 1.h.s. of (A.3) converges when

$$
\sum_{\lambda \in \Lambda} \prod_{1 \leq j \leq n}\left(1+\lambda_{j}\right)^{-3-\left(3-\epsilon_{g}\right)(n-j) \operatorname{Re}(g)-2 \operatorname{Re}\left(g_{1}+g_{2}+g_{3}+g_{4}\right)}<\infty
$$

where $\epsilon_{g}=1$ if $\operatorname{Re}(g) \geq 0$ and $\epsilon_{g}=-1$ if $\operatorname{Re}(g)<0$, which (upon combination with the standard fact that $\sum_{m=1}^{\infty} m^{-\alpha}<\infty$ for $\operatorname{Re}(\alpha)>1$ ) leads us to the conclusion that also for $q=1$ the series on the 1.h.s. of (A.1) converges provided $\operatorname{Re}\left(1+2(n-j) g+g_{1}+g_{2}+g_{3}+g_{4}\right)>0$ for $j=1, \cdots, n$.

\section{Acknowledgments}

The author would like to thank Prof. K. Aomoto for providing copies 
of [Ao, I] and pointing out Ref. [Ma2]. Thanks are due to referee for correcting a mistake in one of the formulas.

\section{References}

[Ao] Aomoto, K., On product formulae for Jackson integrals associated with root systems, Preprint, 1994.

[A] Askey, R., Some basic hypergeometric extensions of integrals of Selberg and Andrews, SIAM J. Math. Anal., 11 (1980), 938-951.

[AW] Askey, R. and Wilson, J., A set of orthogonal polynomials that generalize the Racah coefficients or 6-j symbols, SIAM J. Math. Anal., 10 (1979), 1008-1016.

[B] Bailey, W. N., Series of hypergeometric type which are infinite in both directions, Quart. J. Math., Oxford Ser., 7 (1936), 105-115.

[D] van Diejen, J. F., Properties of some families of hypergeometric orthogonal polynomials in several variables, Trans. Amer. Math. Soc. to appear.

[DS] van Diejen, J. F. and Stokman, J. V., Multivariable $q$-Racah polynomials, Duke Math. $J$. to appear.

[Do] Dougall, J., On Vandermonde's theorem and more general expansions, Proc. Edinburgh Math. Soc., 25 (1906), 114-132.

[E] Evans, R., Multidimensional $q$-beta integrals, SIAM J. Math. Anal., 23 (1992), 758-765.

[GR] Gasper, G. and Rahman, M., Basic hypergeometric series, Encyclopedia Math. Appl. 35, Cambridge University Press, Cambridge, 1990.

[Gu1] Gustafson, R. A., Multilateral summation theorems for ordinary and basic hypergeometric series in $U(n), S I A M$ J. Math. Anal., 18 (1987), 1576-1596.

[Gu2] The Macdonald identities for affine root systems of classical type and hypergeometric series very-well-poised on semisimple Lie algebras, in: Thakare, N. K., Sharma, K. C., and Raghunathan, T. T. (eds.), Ramanujan international symposium on analysis (Pune, India, 1987), pp. 185-224 (Macmillan of India, New Dehli, 1989).

[Gu3] , A generalization of Selberg's beta integral, Bull. Amer. Math. Soc. (N.S.), 22 (1990), 97-105.

[Gu4] , Some $q$-beta and Mellin-Barnes integrals on compact Lie groups and Lie algebras, Trans. Amer. Math. Soc., 341 (1994), 69-119.

[H] Habsieger, L., Une $q$-intégrale de Selberg et de Askey, SIAM J. Math. Anal., 19 (1988), 1475-1489.

[Ho] Holman III, W. J., Summation theorems for hypergeometric series in U(n), SIAM J. Math. Anal., 11 (1980), 523-532.

[I] Ito, M., On a theta product formula for Jackson integrals associated with root systems of rank two, Preprint, 1995.

[K] Kadell, K. W. J., A proof of Askey's conjectured $q$-analogue of Selberg's integral and a conjecture of Morris, SIAM J. Math. Anal., 19 (1988), 969-986.

[Ka] Kaneko, J., q-Selberg integrals and Macdonald polynomials, Ann. Sci. École Norm. Sup. (4), 29 (1996), 583-637.

[Ko] Koornwinder, T. H., Askey-Wilson polynomials for root systems of type $B C$, in: Richards, D. St. P. (ed.), Hypergeometric functions on domains of positivity, Jack polynomials, and applications, pp. 189-204, Contemp. Math., 138, Amer. Math. Soc., Providence., R.I., 1992.

[LM] Lilly, G. M. and Milne, S. C., The $C_{l}$ Bailey transform and Bailey lemma, Constr. Approx., 9 (1993), 473-500.

[Ma1] Macdonald, I. G., Orthogonal polynomials associated with root systems, unpublished manuscript, 1988.

[Ma2] - A formal identity for affine root systems, Preprint, 1996.

[M1] Milne, S. C., A $q$-analog of the ${ }_{5} F_{4}(1)$ summation theorem for hypergeometric series 
well-poised in $S U(n), A d v$. Math., 57 (1985), 14-33.

[M2] Milne, S. C., Basic hypergeometric series very well-poised in U(n), J. Math. Anal. Appl., 122 (1987), 223-256.

[Ro] Rogers, L. J., Third memoir on the expansion of certain infinite products, Proc. London Math. Soc., 26 (1895), 15-32.

[Sch] Schlosser, M., Summation theorems for multidimensional basic hypergeometric series by determinant evaluations, Preprint, 1996.

[W] Wilson, J. A., Some hypergeometric orthogonal polynomials, SIAM J. Math. Anal., $\mathbb{1}$ (1980), 690-701.

Note Added in Proof: $i$. In Theorem 2 it is assumed that the parameters satisfy the genericity condition that $2 \rho_{j}$ and $\rho_{j} \pm \rho_{k}(j<k)$ are nonzero (modulo $2 \pi / i \log q$ ), and that $-g_{r}+\rho_{j}$ and $-g+\rho_{j} \pm \rho_{k}(j<k)$ are not equal to negative integers (modulo $2 \pi / i \log q$ ).

ii. The equality $\Sigma_{\lambda \in \Lambda_{N}} \Delta_{q}(\lambda)=\mathscr{N}_{q, \Lambda_{N}}$ in Theorem 3 holds as a rational identity in $q, t \equiv q^{g}$ and $t_{r} \equiv q^{g_{r}}(r=0, \cdots, 3)$, subject to the relation $t^{n-1} t_{a} t_{b} q^{N}=1$. For $q \rightarrow 1$ the equality degenerates to the identity $\Sigma_{\lambda \in \Lambda_{N}} \Delta_{1}(\lambda)=\mathcal{N}_{1 . \Lambda_{N}}$, which is rational in $g$ and $g_{0}, \cdots, g_{3}$ subject to the relation $(n-1) g+g_{a}+g_{b}+N=0$. 